3.

\title{
Effects of Mobile Platform on Female Engagement in MOBA Games
}

\author{
Gege Gao \& Patrick C. Shih
}

Transactions of the Digital Games Research Association June 2019, Vol 4 No 3, pp 75-116

ISSN 2328-9422

(C) The text of this work is licensed under a Creative

Commons Attribution - NonCommercial -NonDerivative

4.0 License (http://creativecommons.org/licenses/by-nc-nd/ 2.5/).

IMAGES: All images appearing in this work are property of the respective copyright owners, and are not released into the Creative Commons. The respective owners reserve all rights.

\section{ABSTRACT}

Previous research shows that fewer female players participate in competitive games than male players. However, it has been reported that there are more female than male players in King of Glory (KoG), one of the most popular multiplayer online battle arena (MOBA) games on the mobile platform in China. This study aims to investigate how KoG captures the interest of female 
players at a higher level than other games. We compared the game design of KoG with League of Legends (LoL), one of the most popular MOBA games on the PC platform. We followed up with a semi-structured interview study with 20 participants about their gameplay experiences on the two different platforms. Our analysis indicates that mobility, sociability, and lower barrier to entry are the main factors that drove female players to participate in KoG.

\section{Keywords}

multiplayer online battle arena games, MOBA games, competitive games, game design, gender differences, game control, game platform.

\section{INTRODUCTION}

The video game industry has emerged as one of the most popular entertainment platforms in the world, and it continues to draw an increasingly diverse audience in terms of age and gender. Especially in recent years, female participation has gradually increased in the gaming industry. Based on the Entertainment Software Association (ESA) 2017 annual report, 45 percent of US gamers are women (ESA, 2018). However, research shows that female players prefer to play non-violent games, such as social games, puzzle games, board games, etc., while male players prefer violent and competitive games (Hartmann \& Klimmt, 2006; Inkpen et al., 1994; Lucas \& Sherry, 2004; Phan et al., 2012).

This finding is supported by a report from Quantic Foundry (QF). Female participation rates in MOBA games and first-person shooting (FPS) games are only 10 percent and seven percent, respectively, and there is only two percent female participation in sports games (Yee, 2017). Similar results have been found in mobile gaming, especially in Chinese culture where mobile gaming is highly adopted. It has been reported that Chinese female players prefer mobile games, such as Match 3 games, real-time 
strategy games, puzzle games, chess games and music games, over competitive games (QuestMobile, 2018a). Furthermore, male players outnumber female players in certain mobile game genres, such as speed running, action and sports (Umeng, 2018). However, when it comes to King of Glory (KoG) (Tencent Games, 2015), a MOBA game on the mobile platform released by Tencent Games in China (Jiguang, 2017), 54 percent of the players are women. This surprising phenomenon served as our motivation to try to understand what led to such high female participation in KoG in China.

In this paper, we compare KoG with League of Legends (LoL) (Riot Games, 2009) based on interviews of 20 experienced players. Our research objective is to compare KoG with LoL to understand factors that led to higher female participation in a game genre that has been traditionally seen as overly competitive and less inviting to females. As one of the most popular MOBA games in the world, LoL has been well studied. In contrast, there is little systematic research reported on KoG despite it being the most popular MOBA game in China. This study also aims to provide a better understanding about the culture of KoG and how it relates to female gaming participation in China. The findings of this paper not only help introduce Chinese MOBA culture to the general gaming audience, they also encourage game scholars to think about how intercultural differences could offer insights on modern game design. In the next section, we review the literature on gender differences in competitive gameplay and game platform research. We then describe our research methodology, which included semi-structured interviews with players of KoG and LoL. Next, we provide a game design analysis on different platforms and summarize the findings from the interviews. Specifically, we provide detailed comparisons of the similarities and differences between LoL and KoG across dimensions that we identified to be important from the interview study. Finally, we discuss features that could be leveraged to design future games to encourage female participation, especially in multiplayer collaborated competitive games. 


\section{RELATED WORK}

\section{Gender Differences in Competitive Gameplay}

Previous research has found clear differences in video game genre preference across gender. Female players tend to prefer social games, music or dance games, and puzzle games, instead of games with low social interaction and violent content (Hartmann \& Klimmt, 2006; Inkpen et al., 1994; Bonanno \& Kommers, 2005; Lucas \& Sherry, 2004; Phan et al., 2012), whereas men prefer more violent and competitive games (Inkpen et al., 1994; Phan et al., 2012). This is consistent with what QF had found. According to a QF report, only 18.5 percent of the core gamers are females. Consistent with the traditional perception of female gamers, these female core gamers play more casual games such as Match 3 (e.g., Candy Crush Saga, Bejeweled; 69\%), Family/Farm Sim (e.g., Stardew Valley; 69\%), and Casual Puzzle (e.g., Angry Birds; 42\%). What separates them from casual gamers is that they also play Massive Multiplayer Online (MMO) games (e.g., World of Warcraft; 36\%) and MOBA games (e.g., Defense of the Ancients, aka. DOTA, LoL; 10\%) (Yee, 2017). Specifically, in China, it is reported that male players outnumber female players in certain game genres such as speed running, action and sports (Umeng, 2018).

The low female preference and participation in competitive games should be considered in a competitive context. One explanation for female players not showing interest in competitive games could be because they are less interested in the competitive or violent aspects of play (Cassell \& Jenkins, 1998; Hartmann \& Klimmt, 2006; Lucas \& Sherry, 2004). Some researchers claimed that males tend to be more competitive and effective than females in intergroup settings (Cashdan, 1998; Gneezy et al., 2003; Niederle et al., 2007; Vugt et al., 2007). Specifically, Cashdan (1998) claimed that although men and women feel equally competitive, men are more competitive in athletics and for sexual attention while women are more competitive in appearing attractive. 
Schmierbach (2010) mentioned that as "competition serves to reward and reinforce aggressive play” (p. 268), female players, who are less likely to enjoy competition, are less likely to learn violence as "rewards" in competitive play in the same manner as males. However, some research found that the motivation of players determines their game preferences. People play games for different reasons, and even the same game may hold various meanings for different players (Yee, 2006). Based on the survey conducted by QF in 2016, the primary motivations for men to play games are competition (14.1\%), destruction (11.9\%), and completion (10.2\%), while completion (17\%), fantasy (16.2\%) and design (14.5\%) are the primary motivations for women (Yee, 2016). Olson et al. (2008) found that male players use violent video games to express their demands for gaining power as well as their feelings of anger and stress. Hartmann and Klimmt (2006) found that female players prefer games with rich social interaction and dislike "violent content and heavy gender-stereotyping in the presentation of characters" (p. 925). These findings also support the explanations mentioned previously. However, Olson et al. (2008) also found that a considerable number of female players utilize games to express their anger and other emotions. Hartmann and Klimmt (2006) found that social interaction is more important to female players than the gender-stereotyping and violent content in the game. Furthermore, researchers have found that the level of technology integration and dedicated gaming time affects the motivation of female players (Royse et al., 2007; Shaer et al., 2017). Female players who spend a lot of time playing games usually enjoy mastering the games and are motivated by challenges. Competitive games allow them to achieve these goals (Royse et al., 2007; Shaer et al., 2017). For gamers who play mostly casual games, the motivation could be a sense of selfcontrol. Casual gamers still consider themselves "outsiders" and consider masculinity as the prominent factor in competitive games (Royse et al., 2007).

However, some other researchers say the discourse about gender preferences are not about simple binaries, such as violence or 
no violence, but about considering different contexts (Carr, 2005; Jenson \& de Carstell, 2010; Yee, 2008). Game attributes such as graphics, interface, characters and participants' ages, amounts, consumptions and locations could affect a player's gaming preferences. For instance, Yee (2016) found that competition is the most popular motivation among young gamers (ages 13-25), but that category drops to ninth place among gamers who are over 36 years old. Fantasy and completion are the primary motivations for these gamers. Regardless of these sub-contexts, the main hindrance to female players' willingness to play competitive games in a general social context could be the existing genderstereotyping and hostile environment. Previous research has found that female players are often perceived as "outsiders" in the gaming community, and receive both sexual harassment and general harassment due to gender stereotypes (Gray, 2012; Yee, 2014; Nakandala, 2016; Ratan et al., 2015; Taylor et al., 2009; Tang \& Fox, 2016; Shaer et al., 2017; Salter \& Blodgett, 2012). As players become more aggressive in the game world, these stereotypes are progressively internalized and are more likely to be adopted by experts than novices (Bergstrom et al., 2012), which could easily form an unhealthy gaming environment for female players.

Research has found existing gender stereotypes in games (Beasley \& Standley, 2002; Downs \& Smith, 2005; Cassell \& Jenkins, 1998; Taylor, 2009; Mou \& Peng, 2009; Gao et al., 2017; Shaer et al., 2017; Salter \& Blodgett, 2012). Underrepresentation of female heroines, hypersexualized female portraits, and the ways in which hypermasculinity dominated over femininity in game designs not only reinforce existing gender stereotypes (Martey et al., 2014; Brehm, 2013; Todd, 2012; Schröder, 2008), but negatively affects women's perceptions and behaviors of themselves (Richard \& Hoadley, 2013; Vermeulen et al., 2014; Ratan et al., 2015; Shaer et al., 2017) and the overall dominant work culture of the gaming industry (Salter \& Blodgett, 2012; Shaer et al., 2017). 
Different researchers have identified female players with different motivations and preferences in different cultural contexts. However, the majority of gaming research has focused on western countries (e.g., Shaw, 2010) and Japan (e.g., Consalvo, 2016) due to their widespread influence on the global gaming industry. Bjarke and Martin (2016) mentioned that current game studies are centralized on Western Europe and North America, and claimed that this limits the studies across different approaches and perspectives in the interdisciplinarity of game studies. To enrich the game research field, it is necessary for scholars to study the gaming context outside of Europe, North America, and Japan. Chinese game companies, such as Tencent Games and NetEase Games, have released several games that are highly popular among Chinese gamers. Specifically, mobile gaming has become the mainstream among Chinese players due to the wide adoption of mobile devices. In addition, mobile gaming is also one of the approaches to mobile socialization. Both mobile gaming and mobile socialization are nuances of the Chinese gaming culture that are understudied (Liu \& Li, 2011) and gender differences in participation have also not been systematically analyzed as well. Existing reporting of Chinese games mostly involves statistical trends, but does not provide nuanced comparisons that could generate new insights for game research, design, and practices. This paper aims to use KoG as a case of comparison to provide new understanding and research directions in terms of culture and game design. The reasons underlying female players' participation in competitive games are diverse and complex. Researchers are still exploring factors influencing female players' participation in competitive games. Thus, it is difficult for game designers to generate a systematic framework to increase female player participation, especially in different cultural contexts. This paper tries to translate successful Chinese game designs that have led to high female participation into high level design insights for future gaming research. 
Considerable research has been conducted on gaming across different platforms and controllers to understand their effects on the gameplay experience. Researchers have studied player experience and behavior on immersive virtual environments versus traditional platform desktop (Persky \& Blascovich, 2007), and pen and paper platform versus digital platform (Tychsen et al., 2008; Tychsen, 2006). Since few games are developed across different platforms, game companies typically maintain the same game content, but with different game control devices. Therefore, prior research on game input has studied the differences across various controllers, such as keyboards, gamepads, and controllers with different design and functions, and how they affect the playing experience including user enjoyment, motivation, engagement, and social behaviors (Birk \& Mandryk, 2013; Brown et al., 2015; Gerling et al., 2011; Lucas \& Sherry, 2004; Tychsen, 2006; Limperos et al., 2011; McEwan et al., 2012; Rogers et al., 2015). As more platforms have become available to the public, gaming companies have realized the importance of developing games across different platforms. This paper aims to contribute to game research on gaming across different platforms such as PC, mobile phones, and consoles. No prior research has been conducted on game design to explore how different platforms affect female participation in MOBA games. One of the goals of this paper is to inspire other researchers on studies between game platforms and female participation.

\section{METHODS}

\section{Game Design Comparison}

We conducted a design analysis that focused on unpacking the differences in MOBA game design on mobile versus PC platforms. We chose to study KoG mainly because it is a popular mobile MOBA game with a high level of female participation in China. 
We wanted to understand and explore how KoG successfully attracted female participation. Since LoL is a popular MOBA game on PC that is known for its low female participation and an environment toxic to female participants, the contrast with KoG could reveal factors that could contribute to female inclusion. Beyond that, these two games are also similar in the following ways: 1) The fundamental game mechanics and design elements are very similar; 2) they are the most popular MOBA games on mobile and PC platforms; 3) they are owned and developed by the same game developers.

\section{MOBA Games}

MOBA games, as a genre, were originally derived from the Aeon of Strife map in StarCraft. Later, Blizzard Entertainment released Warcraft 3 (Blizzard Entertainment, 2002) that included a mod called Defense of the Ancients (DOTA) that popularized the MOBA genre. Later, the emergence of LoL and DOTA2 gradually formed a stable map mode and game mechanics of MOBA games. A MOBA game typically contains the following map layout and game mechanics: two teams with five players each located on opposite ends of the map with the goal of destroying the nexus located in the enemy's base. Three lanes (top, mid and bottom) connect the two bases, and two turrets are located on each lane for each team. Players need to destroy all of the turrets on all lanes to approach the nexus, for which players need to slay enemies and non-player characters (NPC) such as minions and monsters to level up, get buffs (a buff provides a status uplift effect in the game), and earn money to buy necessary equipment from the in-game store.

Players usually fill different roles on different positions. For example, the top role is mainly for guarding the top lane, and it usually requires avatars with high defense and/or damage ability, a.k.a. "Tank." The in-game avatars are called champions, and there is a wide range of champions to select from in MOBA games. These champions are designed with distinct appearance, abilities, and fantasy backstories, which allows players to form various 
team compositions in the games, depending on their preference and strategy. Although most MOBA games are released on the PC platform, since 2013 more and more MOBA games are being released on mobile platforms. The earliest mobile MOBA games, such as Solstice Arena (Zynga, 2013) and Vainglory (Super Evil Megacorp, 2014), only supported 3 vs. 3 mode and with abstract maps, compared to the maps on PCs. Later, KoG and Mobile Legends (Moonton, 2016) started to support 5 vs. 5 mode as their main game mode. The complexity of the maps in these games is pretty close to the maps played on PCs. The 5 vs. 5 mode has become the typical and main game mode in MOBA games on mobile platforms. Vainglory started to support 5 vs. 5 mode this year to attract more players.

\section{King of Glory}

King of Glory (or 王者荣耀 in Chinese) is a multiplayer online battle arena (MOBA) game on the mobile platform in China developed by Tencent Games, which is a subsidiary of Tencent (King of Glory, 2015). In July 2017, it was reported that the game had more than 54 million daily active players and 163 million monthly active players, according to Jiguang, an IDG Capitalbacked big data platform (Jiguang, 2017). Specifically, it was reported that females made up 54.1 percent of the overall players, outnumbering male players for the first time in any MOBA game (Jiguang, 2017). KoG is a new and highly popular mobile MOBA game in China that is understudied. The goal of this research is to introduce $\mathrm{KoG}$ to a broader audience, and to uncover factors that encourage female players to participate in competitive MOBA games such as KoG.

\section{League of Legends}

League of Legends (LoL) is a MOBA game on the PC platform developed by Riot Games, which is a subsidiary of Tencent Games. It is one of the most played video games on the PC platform in the world with more than 27 million daily active 
players and 67 million monthly active players (LoL, 2017). Female participation was reported to be 35 percent in 2017 (Bloomberg, 2017). Besides the classic 5 vs. 5 mode, LoL also provides other play modes, such as All Random All Mid, Bot, and The Twisted Treeline (3 vs. 3) for players to enjoy different playing experiences. In this work, it is our goal to compare and contrast player experiences on KoG and LoL to understand the difference in female participation in these games given that, since they are developed by the same company, they share very similar map layouts and game mechanics.

\section{Semi-structured Interviews}

We conducted semi-structured interviews with 20 Chinese participants (10 women, 10 men) who were recruited by the researchers on game events and conventions in the US from JuneDecember 2017. To obtain more meaningful data and reduce gender bias, the researchers recruited women and men in equal numbers, and ensured all participants had at least a half year of playing experience on at least one platform. The interviews were conducted in Chinese and in person, with consent to audio record. The recorded interviews were transcribed and translated into English by the first author. All participants participated voluntarily with no compensation. The interview protocol was adapted from the authors' prior research on MOBA games (Gao et al., 2017) with a particular focus on topics such as what game the participants mainly play, what motivated them to participate in these games, what in-game role they usually play, how they communicate with other players, etc. Table 1 details the demographic information for all participants. Table 2 shows the gender distribution regarding their game platform experiences.

\section{Interview Data Analysis}

We applied open coding (Corbin \& Strauss, 2015) to the translated transcripts. The authors discussed the initial codes. Chinese gaming culture was considered during the coding process to 
86 Effects of Mobile Platform on Female Engagement in MOBA Games

identify the data more accurately. For example, we identified and transcribed Chinese gaming slang, such as “开黑” (friends who play together) “腿短” (champions with a small area of effect), and others based on popular Chinese gaming culture. An affinity diagram (Holtzblatt \& Jones, 1993) was used to organize the open codes to iteratively refine emerged themes.

\begin{tabular}{|c|c|c|c|}
\hline Participant code & Gender & Age range & Education level \\
\hline P1, P5, P6, P16, P17, P18 & M & $21-28$ & Master \\
\hline P2, P3, P4, P15 & M & $24-26$ & Bachelor \\
\hline P12, P20 & F & 24 & Doctoral \\
\hline P7, P8, P11, P13, P14 & F & $23-28$ & Master \\
\hline P9, P19 & F & $24-27$ & Bachelor \\
\hline P10 & F & 20 & Undergrad \\
\hline
\end{tabular}

Table 1: Participant Demographics.

\begin{tabular}{|c|c|c|}
\hline Platforms & Women & Men \\
\hline Both & 2 & 6 \\
\hline Mobile Only & 6 & 2 \\
\hline PC Only & 2 & 2 \\
\hline
\end{tabular}

Table 2: Gender distribution regarding playing experience on different platforms.

\section{GAME DESIGN ANALYSIS}

In this section, we compare the game designs of KoG and LoL in terms of avatar design, gameplay design, and social interaction design, as well as tutorial design. 


\section{Avatar Design}

Avatars, also known as champions, are the core component in MOBA games. Champions feature different appearances, backgrounds, and capabilities, and they give players a wide range of options in the game. At the time of the writing of this paper, there were 141 champions in LoL, including 46 women (32.6\%), 94 men (66.7\%), and one dual-gender champion (Kindred) (LoL, 2018), and there were 84 champions in KoG, including 23 women (27.4\%) and 61 men (72.6\%) (KoG, 2018). Although champion gender distribution between LoL and KoG was similar, most of the champions in KoG were depicted based on well-known Chinese historical figures or fictional novel characters. For example, champions such as Daqiao (Chinese: 大乔), Xiaoqiao (Chinese: 小乔), and LüBu (Chinese: 吕布) are historical figures from the Three Kingdoms period (220-280 AD) who were documented in the Records of the Three Kingdoms (Chinese : 三国志). Champions such as 哪吒 and 姜子牙 are fictional characters in Investiture of the Gods (Chinese: 封神演义), a fantasy novel written during the Ming dynasty (1368-1644).

\section{Gameplay Design}

Different platforms require different methods of input. A keyboard and a mouse are required for input on the PC platform, while the touch screen is the input method for the mobile platform. Figure 1 shows the input control method for KoG on a smartphone (KoG, 2018). A virtual joystick is created for the left thumb to control the avatar movement, and clickable virtual buttons are designed for the right thumb to attack and cast spells, or use abilities. LoL requires the combination of a keyboard and a mouse. The mouse is used to control avatar movement, and select items, and keys such as Q, W, E, R, and other number keys are used to attack, cast spells/use abilities, and consume or activate items in the inventory (LoL, 2018). (See Figure 2) 


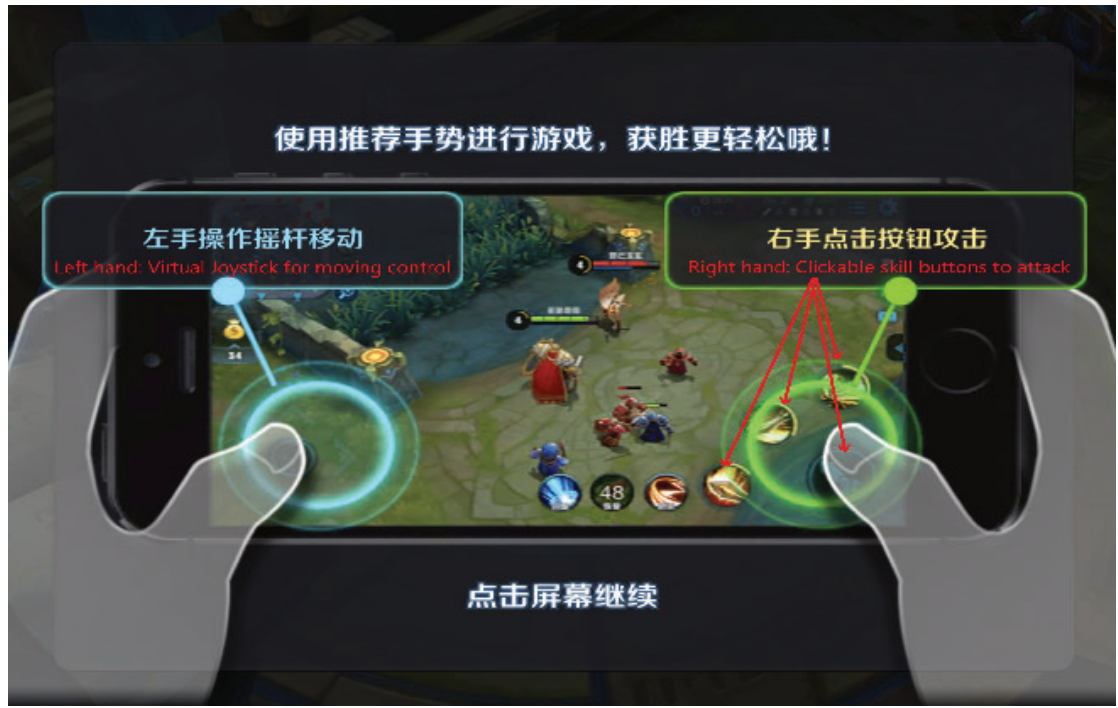

Figure 1: The input control method for KoG.

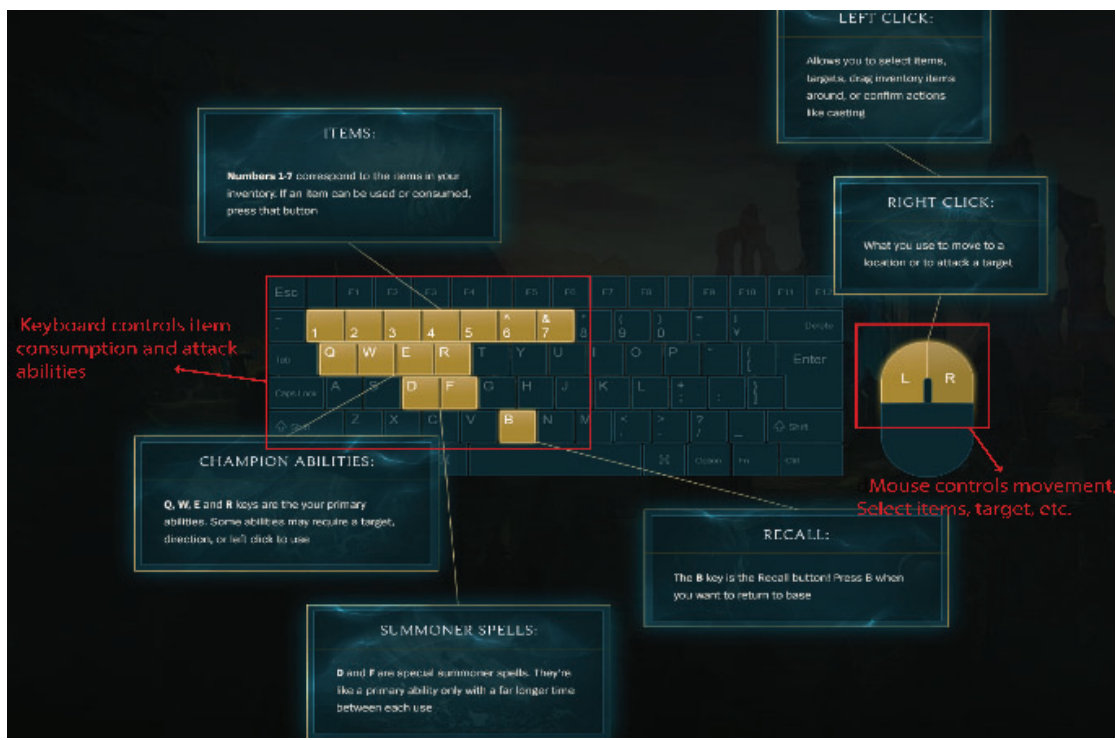

Figure 2: The input control method for LoL

Besides the differences between input control methods, several game mechanics that are common in MOBA games on PC platforms such as LoL have been adapted for KoG to be more playable on the mobile platform. First, all players have full vision 
of the map, which means players don't need to place wards and fight to gain vision of the map during the game. However, KoG provides another mode called the "fog mode," in which players could experience the fog of war just like in LoL. Second, the number of abilities of each champion is reduced from five in LoL to four in $\mathrm{KoG}$, and the required level to learn the most powerful skill of a given avatar is reduced from Level 6 to Level 4 .

\section{Social Interaction Design}

While LoL players need to register for accounts to $\log$ in, and must either acquire other players' IDs to add them as in-game friends, or add friends by linking the game accounts to social media accounts (see Figure 3(a)), KoG has omitted these tedious tasks by allowing players to log in using either a QQ account or a WeChat account. QQ and WeChat are the two most popular instant messaging applications in China, both of which are developed by Tencent. Thus, players can communicate and play with their friends on social media immediately after logging in, and they can send daily gifts to their friends, and invite their friends to play together (see Figure 3(b)) (KoG, 2018). Additionally, KoG incorporates the mentoring and relationship systems of massive multiplayer online role-playing games (MMORPG). In the mentoring system, an experienced player could join forces with a new player to help new players quickly become more familiar with the game, and gain experience and rewards by completing specific tasks together. In the relationship system, players could build different relationships, such as a "romantic partnership" or "close friendship," with other players, based on their closeness in the game. Special game effects will appear in the game when they play together. 
90 Effects of Mobile Platform on Female Engagement in MOBA Games

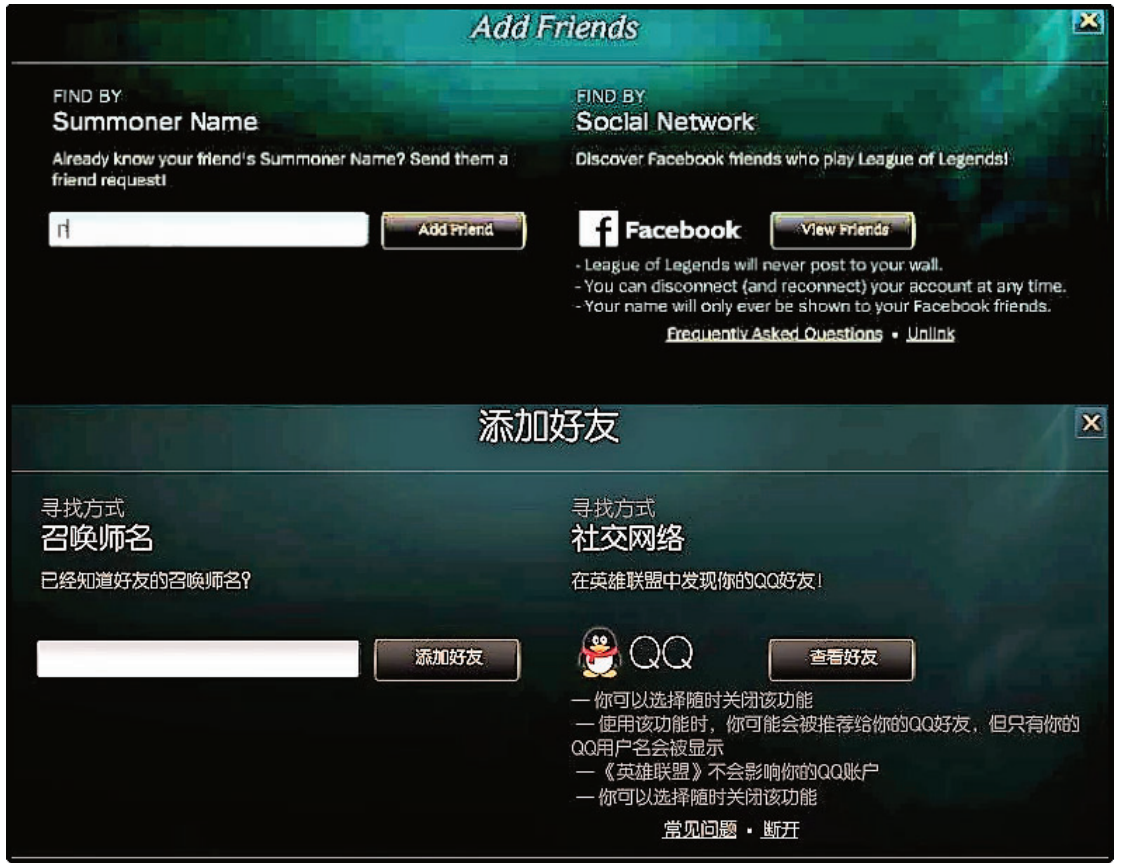

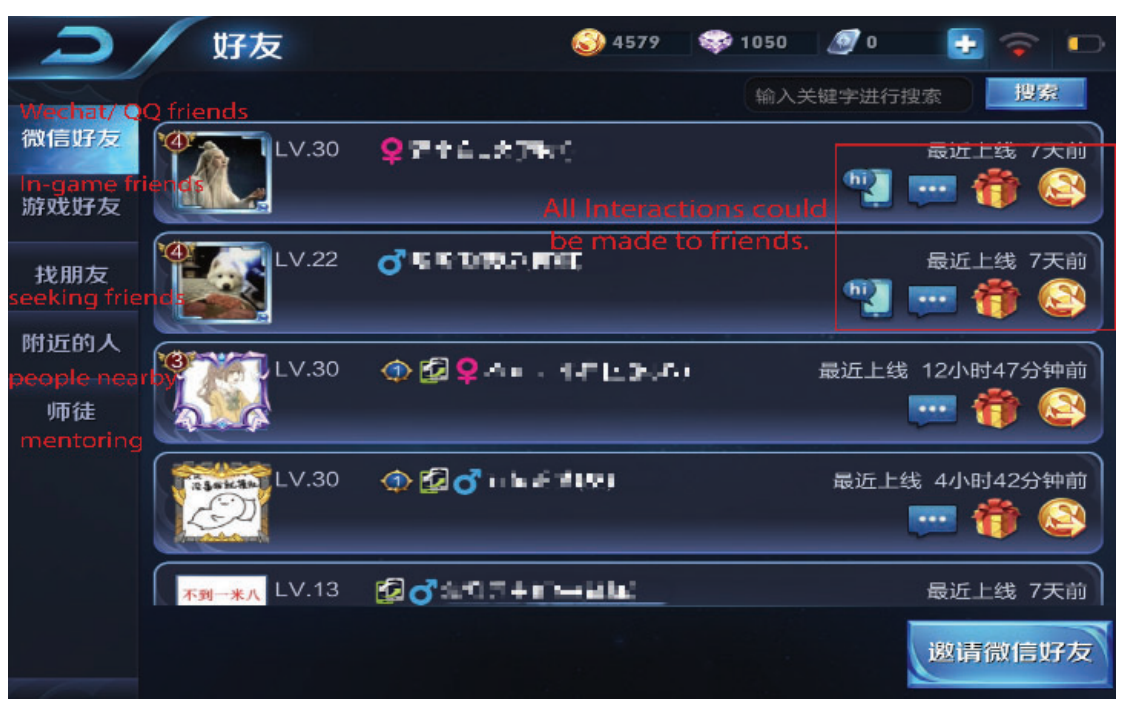

Figure 3: Interface of the Friends tab: (a) in LoL (top: in English and Chinese); (b) in KoG (bottom). 
LoL provides complete tutorials in which players are introduced to all necessary information at the beginning of the game. The tutorials include a basic tutorial and battle training (see Figure 4(a)). The basic tutorial teaches the fundamentals of LoL, such as how to move and how to attack, and battle training teaches advanced skills such as how to ambush and get jungle buffs. Players can repeat these tutorials to gain more experience. The tutorials usually use pictures and videos to illustrate the gameplay in both LoL and KoG (see Figure 4(b) as example). Different from LoL, KoG provides a series of interactive tutorials for new players to learn and practice (see Figure 5(a)) (KoG, 2018). The tutorials are presented in multiple sections. Players can focus on certain skills by repeating the specific section instead of going through the whole tutorial. In addition, KoG provides an incremental reward task structure that helps them become familiar with the game mechanics and environment, with careful scaffolding (see Figure 5(b)) (KoG, 2018).

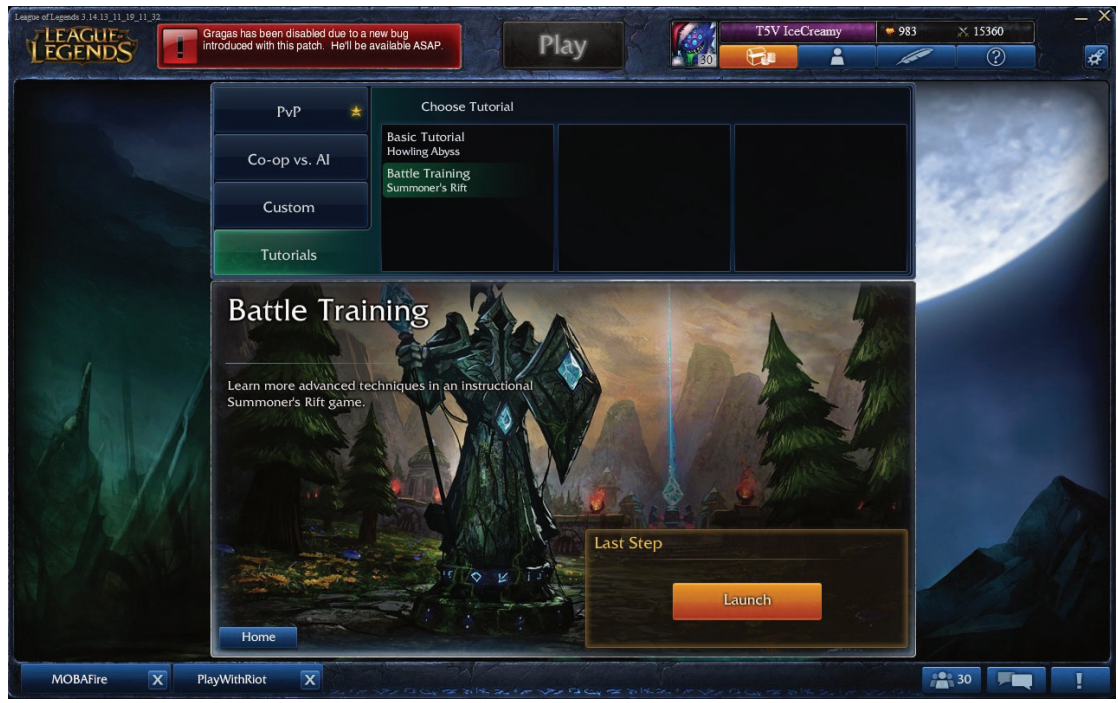


92 Effects of Mobile Platform on Female Engagement in MOBA Games

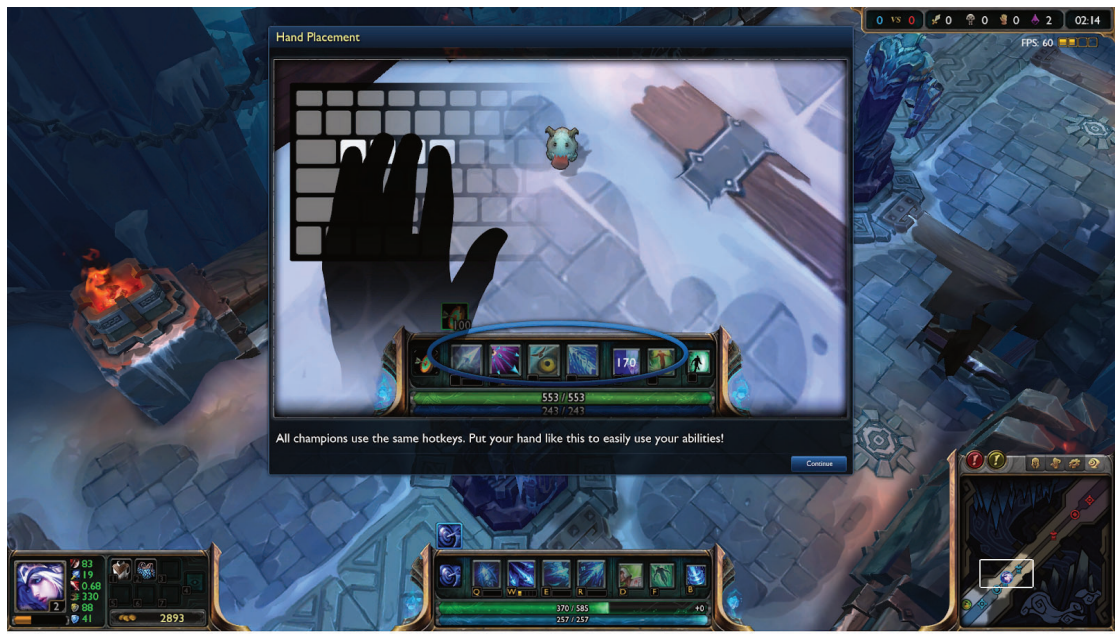

Figure 4: (a) Two training tutorials in LoL (top);(b) gameplay instruction in the tutorial of LoL (bottom).

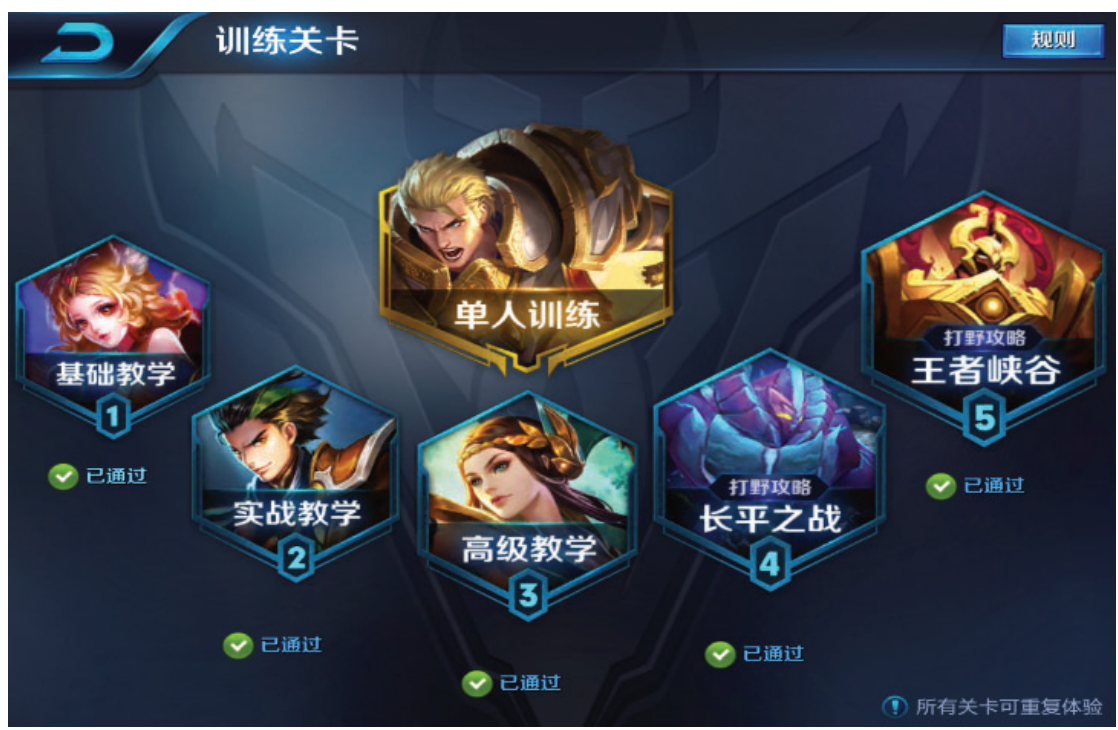


Effects of Mobile Platform on Female Engagement in MOBA Games 93

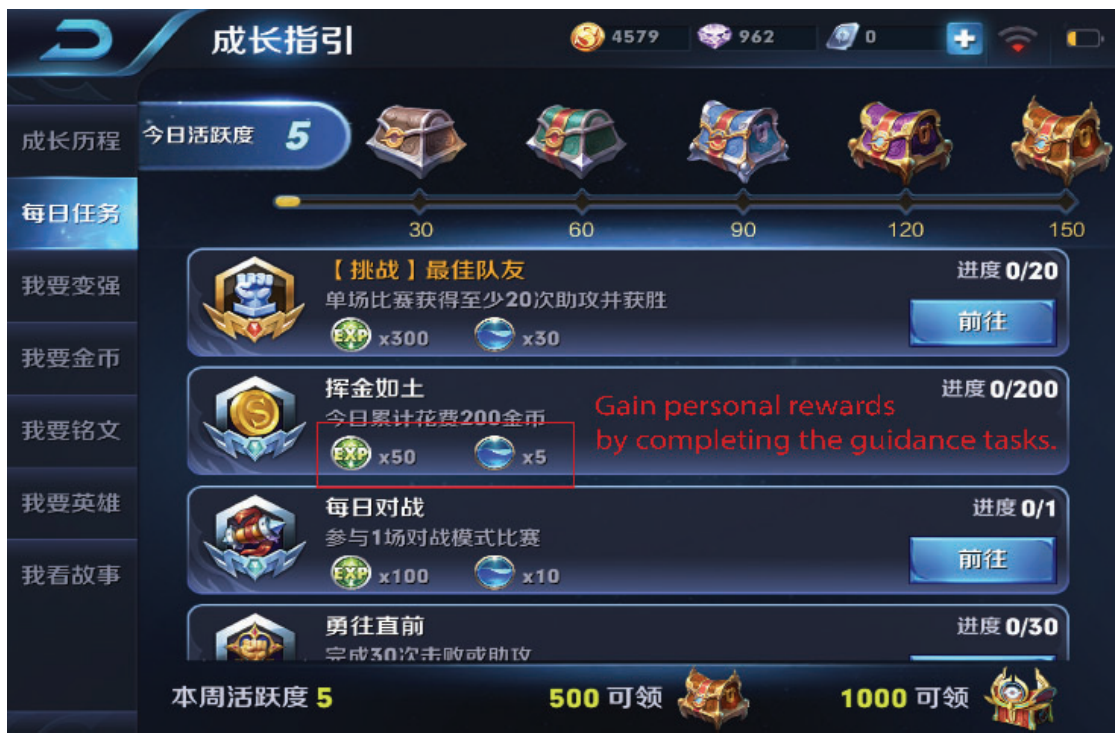

Figure 5: (a) Series of interactive tutorial in KoG (top);

(b) incremental reward task structure in KoG (bottom).

\section{FINDINGS}

Based on our analysis of game design and interview transcripts, we identified the lower barrier to entry, mobility, sociability, and avatar perception as key factors that drove people to participate in KoG. Additionally, we compared the interview results in terms of gender differences and tried to explore the connection between these factors and gender.

\section{Lower Barrier to Entry}

As opposed to casual games, MOBA games typically feature a steep learning curve at the beginning (Gao et al., 2017). It usually requires a lot of time dedication and discipline from the playerbase. In order to win, players usually practice a lot and spend large amounts of time on the game. Thus, it is very difficult for new players to get comfortable with the game. 


\section{Design for casual gamers}

By reducing the difficulty level of the gameplay and input control, as described in the Gameplay Design section, Tencent Game developers made the game easier for players to learn. These changes also reduced the time required to play one round of KoG. In our interviews, all participants mentioned that KoG was very easy to learn, the game control was simple and intuitive, and it had a shallow learning curve. Participants also reported that the interactive tutorial series, the incremental reward task structure, and the mentoring system made KoG easy to learn for the new players.

P2 (M, 24): “KoG is easy because the virtual joystick is highly fault-tolerant, which could provide a good gaming experience to most players."

P6 (M, 28): "I think the mobile gameplay is very convenient and simple compared to PC. KoG also requires fewer playing skills and strategy, and is friendly to new players."

$P 7$ (F, 23): "KoG is much easier than LoL. It enlarges the cast area of effect of champions and provides functional tutorials for new players. Therefore, new players could get in very fast."

P10 (F, 20): “My friends all recommended KoG to me. I was not interested in MOBA games before, (but) since $\mathrm{KoG}$ is much easier to control and play, I tried and found it pretty interesting."

Six out of eight female participants that we interviewed were novice MOBA players, whereas six out of eight male participants were experienced MOBA players. While we only interviewed 20 participants, these findings are consistent with the statistical trend that KoG is attracting many more new female gamers to play the game. 


\section{Frustration in certain serious gamers}

Although KoG lowered the entry barrier for mobile players to broaden its audience, it frustrated players who preferred the traditional style of MOBA games. For instance, there were four participants (two women, two men) who chose to play MOBA games (i.e., LoL, DOTA) only on the PC platform. When asked what prevented them from playing KoG, they provided the following rationales:

$P 13$ (F, 27): "I do not play KoG because it is not fun to me. I like to play LoL because it requires more serious strategy and teamwork. I also think the screen on the mobile phone is too small for MOBA games to gain complete control."

P16 (M, 27): “I don't play KoG mainly because there are too many terrible teammates. Since KoG decreased the gameplay difficulty, a lot of players only play for fun. They don't care about the teamwork or win rate, which destroys my playing experience completely."

We also found that the demand for game complexity and the attitude towards gameplay varied among male and female players playing on the PC versus mobile platforms. Some of our participants made the following comments:

P2 (M, 24): "Although KoG has more players and gross than LoL, it can't take LoL down. They have different target users. LoL is more about strategy and teamwork whereas KoG is for social and entertainment."

P4 (M, 25): “I think KoG can't replace LoL because they have different target users. LoL players usually those who have more time to play, and KoG is opposite. 
I could gain more self-gratification in long-time playing."

P19 (F, 24): “KoG might take away some market share from LoL, but it will not replace LoL. It is because users have different needs, so these two games would have their own core users and market."

Our research supports previous findings that players have different motivations toward gaming, especially among players with different levels of time dedications (Hartmann \& Klimmt, 2006; Royse et al., 2007; Yee, 2006; Shaer et al., 2017; Yee, 2016). Serious gamers (people who dedicate much time to playing noncasual games) such as P13 (F, 27) and P16 (M, 27), usually demand games that present significant difficulties and challenges. In our findings, serious gamers, both female and male, wanted the game to be complex and wanted their teammates to be dependable. However, most casual gamers play games to pass time or to socialize with other gamers, so they want the games to be less difficult and time consuming. Therefore, for serious gamers, mobile platforms might not be a good platform for them to play. Our findings also support the result of previous research that found female players who spent more time on gaming were more likely to play on a computer or a console than on a mobile phone (Shaer et al., 2017).

KoG developers made a conscious decision to sacrifice complexity for playability on mobile devices to broaden its appeal to the public. Such a tradeoff is considered to be positive for both female and male players who prefer social and casual games, which require less time dedication, although female players, being the majority, are likely to be more affected. However, it may be less desirable to serious gamers, who prefer more complex games that are more demanding. 
Smartphones are highly portable, and are carried by most people at all times. Mobile games, particularly casual games, are often used to pass time (e.g., while commuting on public transport). ESA reports that Americans spend one-third of their commuting time playing games on mobile phones and tablets (ESA, 2016). Therefore, designers of mobile games must take into account the ever-changing context and mobile nature of the environment. In adapting LoL on PC to KoG to the mobile platform, the game design removed some features to reduce the total time cost for each round of the game to accommodate the mobility aspect of mobile platforms. Our female participants indicated that the mobile nature of smartphones also makes it easier for them to play without the limitations of location and device hardware that PC gamers usually face.

P8 (F, 23): "I like KoG because it takes less time for each match. It is not like PC games, which take a long time, and it has less delays."

P13 (F, 27): "The mobile game requires less time, which is good for killing time. LoL takes a longer time commitment to set up and play, so it is more difficult to get into the game when you're constantly on the go."

P19 (F, 24): "It takes less time to play one round in $\mathrm{KoG}$, and it is also very convenient to play using a smartphone because it's always with me."

The mobile feature also makes it possible for people to meet up and play the game together in a collocated fashion. This collocation would facilitate the frequency and quality of communication between players, which leads to higher team performance (Huffaker et al., 2009). Both the male and female participants mentioned that they prefer face-to-face 
communication while playing, so they usually gather together first before beginning play.

P3 (M, 26): "I usually communicate with my teammates face to face, and we usually meet first then play together."

P4 (M, 25): "I play with my friends most of the time, and we always communicate offline.”

P10 (F, 20): "I usually play with my friends if we meet in person."

P12 (F, 24): “It is really fun to sit next to my friends and play KoG together."

PC platforms usually face many challenges regarding team communication. For example, verbal communications are usually accomplished through text and voice chat. However, text chat is distracting in time-limited competitive games (Innocent \& Haines, 2007), and players might find it difficult to type while controlling the avatars. Despite occasionally poor voice connection quality, voice chat sometimes causes players confusion over who is talking (Halloran et al., 2004), especially when mixed with game sounds (i.e. background music, sound effects).

Although the mobile platform provides more mobility, convenience, and communication to players, it causes other unintended consequences that could negatively impact the gameplay experience. Our participants mentioned that people dropped offline on the mobile platform more frequently than in traditional MOBA games like LoL.

P5 (M, 27): “There are always people who would drop offline intentionally. Some of them might've encountered a network problem, but some others just quit during the game because they have to go. It is much more common to quit on the mobile phone." 
P6 ( $M, 28):$ "I know there are some players who drop offline due to being interrupted by friends, poor network connection, incoming calls, and battery issues.”

To avoid this problem, P9 uses the tablet to create a better gaming environment for KoG:

P9 (F, 26): “I usually play on my tablet, because sometimes there are incoming calls to my mobile phone while I am playing, which is annoying. In addition, sometimes the network is poor on my mobile phone, and the WiFi connection on my tablet is much more stable."

The mobile nature of smartphones makes it more convenient for players to play a quick round of KoG while being on the go, which makes it more attractive for casual gameplay and for many female players. However, connectivity, hardware, and interruption issues could all affect the gameplay experience of all players in significant ways.

\section{Sociability}

Sociability is a key, if not the most important, feature when playing multiplayer games. ESA reports that Americans are increasingly relying on multiplayer games as a means to socialize with their friends, family, and spouse (ESA, 2018). It reveals a high preference for social gaming by Americans, with 55 percent of the most frequent gamers in the US believing video games help them connect with their friends, and 46 percent believe it helps their family spend time together. Specifically, people in China have formed a lifestyle of mobile socialization, and 10.5 percent of the mobile social time was accomplished through mobile gaming (QuestMobile, 2018b). Prior research found that both female players (Hartmann \& Klimmt, 2006; Inkpen et al., 1994; Lucas \& Sherry, 2004; Phan et al., 2012; Royse et al., 2007) and male 
players (Olson et al., 2008; Yee, 2006) prefer games with the support of social features. Specifically, in Royse et al.'s (2007) research, female players consider social features more important than violent and gender-stereotyping gaming features.

\section{Socially-focused Play in Friend Circle}

KoG supports social interaction by leveraging social media account logins to tap into the player's existing social network. It provides random game matches between friends, allowing players to choose to play with friends or strangers. In addition, it incorporates social interaction design features, such as a mentoring system and relationship system, to encourage communication, interaction, and coordination among players. Furthermore, KoG provides periodical milestones in each round of matches, such as MVP and Penta Kills, that can be shared on social media (e.g., moments in Wechat). KoG can form a competitive mechanism between a circle of friends and a gamer circle, which facilitates the interpersonal spread of the game. These social features not only enable gameplay between close friends, but possibly strengthens the connection between social acquaintances when games are played together.

About 90 percent of the participants in our study played MOBA games with friends, and those who only played KoG (six women, two men) reported that they were introduced to playing KoG because most of their friends were playing it. Specifically, both of the female and male participants regard it as a social game, which helps them hang out with their real friends.

P1 (M, 21): "I play KoG for social reasons; it is interesting only if I play with the people I am familiar with.”

P5 (M, 27): “I play KoG mainly because I can play with many friends, which is fun." 
$P 7$ (F, 23): “I think KoG is really good for socializing, I play it with my roommates all the time at first, then I play with my friends who I haven't been in touch with in a long time."

P9 (F, 26): "I usually play with my friends. We usually play when we hang out together."

P19 (F, 24): “I think KoG is a social method. For example, friends could play together while waiting to eat in a restaurant. It could get a lot of social-driven players like me."

Although we cannot claim that female players use KoG as a way to socialize more than male players, social play with real friends is especially attractive for novice female players who are new to the game. These novice players are casual gamers who prefer casual games and puzzle games, or have very little gaming experience. While many started playing KoG to socialize with their friends, it is possible for them to gain interest and become serious gamers over time. It is also possible that some female players are "competitive game neophobic", meaning that they fear playing competitive games due to the gender stereotypes (Richard \& Hoadley, 2013; Vermeulen et al., 2014; Ratan et al., 2015; Shaer et al., 2017). As the female participation in KoG rises, more serious female gamers may emerge due to the equalizing number of both sexes in the game.

\section{Less in-game conversations with strangers}

Strong sociability means more interaction and communication, which has some side effects, and "toxicity" is one of them. Prior research claimed that the gaming environment is especially toxic for women (Gray, 2012; Yee, 2014; Nakandala, 2016; Ratan et al., 2015; Taylor et al., 2009; Tang \& Fox, 2016; Shaer et al., 2017; Salter \& Blodgett, 2012). The toxicity usually includes aggressiveness, hostility, offensive verbal attacks, and trash talk 
from strangers over voice or text chat, which forced many female players to quit the game (Salter \& Blodgett, 2012; Shaer et al., 2017). Our findings show that playing KoG on mobile devices can reduce the in-game conversations, which in turn reduces toxic encounters. Our female participants provided several reasons that explain the lower level of toxicity in KoG. In LoL, people could type via keyboards. In KoG, in addition to being collocated with friends, people could also choose in-game chat, either in text or voice. However, it is more difficult to type using the touchscreen because KoG is played on mobile devices. In LoL, people usually play in private places because it is played on PCs. The environment allows them to communicate with their voice using chatting software, such as Skype and Discord. In KoG, people tend to play in public areas in the presence of other people who might not be playing the game (e.g., during school lunch breaks, work breaks, etc.). Playing in the presence of others makes using voice chat less convenient. In addition, similar to the voice chat in LoL, KoG's voice chat can also be prone to poor voice quality.

P11 (F, 24): "My hands are always busy playing, so I don't have the time for typing in the game. Also, I sometimes play KoG during my work break, I don't want other people know I'm playing..."

P12 (F, 24): “Many people play KoG in public places, such as work break, instead of gaming environment or private space, then it is not proper to do voice chat."

P20 (F, 26): “I think the gaming environment of both LoL and KoG are getting better. LoL probably because less people are playing it, and KoG is due to the inconvenience of in-game communication."

Our female participants also reported that the complexity of KoG is less that of LoL, which makes it less necessary to communicate during gameplay. 
P11 (F, 26): "I heard from others that LoL is very difficult, which needs more communication during the game, no matter texts or pings... So I only play KoG."

P19 (F, 24): "I usually play with my friends, and I don't usually communicate when I play alone. I don't think it is necessary except you are playing a top-ranking match just like the matches in LoL.”

P20 (F, 24): “It is easier than LoL, so there's no need to communicate when I'm playing with strangers."

With less in-game communication, there is less chance of encountering strangers who treat female gamers with hostility. The lack of in-game communication also makes it easier to conceal one's gender during the game. Five out of eight male participants who play KoG reported that they couldn't figure out the gender identity of other gamers, although P1, P2 and P6 expressed that they could infer the gender from a player's in-game ID and profile photo.

The ability of Tencent Games to turn a competitive MOBA game like LoL into a more social game is instrumental in encouraging female participation in KoG. It not only encourages female players to play with their friends, but affords female players a less toxic gaming environment.

\section{Avatar Perception}

Previous research has found that avatar appearance affects players' in-game performance (Peña et al., 2009; Yee \& Bailenson, 2007; Gao et al., 2017). The champions of LoL are designed in a fictional fantasy context. As previously mentioned, the avatar design in KoG is based on well-known historical figures or fictional characters in fantasy novels, which might encourage people to play due to their familiarity with the characters. Based on a report from Penguin Intelligence (PI), which is a research institute owned 
104 Effects of Mobile Platform on Female Engagement in MOBA Games

by Tencent, female players in KoG care more about champion appearance than male players, which is consistent with previous research results (Gao et al., 2017). Male players in KoG care more about historical context of the champions than female players (PI, 2017).

In our study, two female participants (P8, P20) and one male participant (P17) mentioned that they wanted to play the game because they liked the aesthetics of the champions in KoG. Besides aesthetics, two of our male and female participants (P8, P18) mentioned that they were attracted by the Chinese historical contexts of the champions. Although we interviewed only 20 participants, these findings could help explain why KoG is popular among Chinese gamers.

P8 (F, 23): "I like KoG because the champions are relevant to the Chinese culture and are very goodlooking."

P20 (F, 24): "I like the champions in KoG because they are adorable, and both the skins and the skill effects are amazing."

P17 (M, 22): “The reason that I started to play KoG was that my friends had posted the pictures of Xiaoqiao (Chinese name: 小乔) and Daqiao (Chinese name: 大 乔) (female champions in KoG) on Wechat, and I think they are so good-looking."

P18 ( $M, 23):$ "The champions in KoG, are based on historical figures, which make me feel at home."

Avatars generated from well-known historical figures establish a connection between avatars and players. This connection might result in a more comfortable playing environment, which attracts people to play. Moreover, the avatar appearance influences players as well. Avatars designed with high aesthetics are more likely to attract both female and male players to play in China. 
This paper explores the main factors that drive female players to participate in KoG, in a study that involved game design analysis and semi-structured interviews of 20 experienced MOBA players. We provide an analysis of the game in terms of gaming mode, game design, and the gaming culture, and draw comparisons with LoL. We also provide a general understanding of KoG that has not been previously reported in gaming literature. Based on our analyses, a lower barrier to entry, mobility, sociability, and avatar perception are the main factors that led to the increase in female participation in KoG. The lower barrier to enter KoG results from less time dedication and lower level of complexity. Novice players would not continuously get frustrated in the course of learning the game and by losing games to experienced players. Therefore, game designers should consider adjusting the "deepness" of the game to attract more players. To avoid frustrating serious gamers, game designers should also consider adding features that have an element of randomness, to appeal to more players.

Recently, online multiplayer Battle Royale games, such as PlayerUnknown's Battlegrounds (Bluehole, 2017) and Fortnite (Epic Games, 2017), have become extremely popular. This kind of game, which is packed with a high degree of randomness, can increase the possibility of wins for novice gamers, as well as bring some uncertainty, fun, and tension to the game. Therefore, it has the potential to attract more players to participate in the game.

By comparing the responses of female and male participants, we found that female players are more likely to be attracted by the mobility and sociability in KoG, compared to male players. Females feel comfortable playing KoG because they encountered less trash talking and hostility, unlike LoL. Female players are more likely to be introduced to a game and to play with their friends using mobile devices. Serious female players, however, might focus on PC platforms for high gaming complexity. Therefore, game designers should consider the features of different 
platforms during the design process. For games on PC platforms, as the mobility is limited, game designers could focus on improving the sociability of games by providing more social interaction functions for players, such as the mentoring and relationship systems in KoG. Furthermore, PC games could also provide more game modes at different levels of difficulty. Less gaming complexity not only lowers the barrier of entry for novice female players, but also reduces unnecessary in-game communication with strangers. Lessened communication with strangers also allows female gamers to more easily conceal their identity, which decreases the toxicity during gameplay. Moreover, game designers could also make efforts to improve avatar designs. Avatars with appealing aesthetics and backgrounds that people are familiar with are more likely to engage female players. For games on mobile platforms, besides maintaining the current mobility and sociability, game designers should also consider offline problems, such as low battery and poor network connections to enhance experiences for players. Beyond that, game designers should consider player motivation and the factors uncovered in this study to create more socially inclusive games. The design considerations proposed in this study are essential for making games more friendly and inviting to female players. This study not only introduces $\mathrm{KoG}$, a popular mobile MOBA game in China, to a wider audience, but reveals possible factors affecting female participation in MOBA games and casts light on new research directions. The findings of this research could inform game design in other game genres that have similarly low female participation rates, especially in multiplayer competitive team-based games.

There are some limitations in this study. First, we only involved 20 participants; future studies could involve more participants to confirm and generalize the findings. In terms of demographics, our participants were all under 30 years of age, which means the findings of this study may not apply to older gamers. Furthermore, we did not account for factors such as time availability to play video games. The ability to play a quick game on the go on mobile 
devices may be even more important for people who may have less time to play video games.

Recently, Tencent released the international version of KoG called Arena of Valor (AoV) (Tencent Games, 2018), previously called Strike of Kings. The AoV avatar designs and gameplay are different from KoG. Future studies could focus on comparing AoV with KoG and LoL to unpack differences in cultural influence and how game design could impact female participation at the international level. Furthermore, AoV has also been released on Switch, a video game system developed by Nintendo. Therefore, future studies will be conducted exploring other user-related design features on more diverse platforms.

\section{BIBLIOGRAPHY}

Beasley, Berrin, and Tracy Collins Standley. "Shirts vs. skins: Clothing as an indicator of gender role stereotyping in video games.” In Mass Communication \& Societyvol. 5, no. 3(2002): 279-293.

Bergstrom, K., J. Jenson, and S. de Castell. "What's 'choice' got to do with it? avatar selection differences between novice and expert players of World of Warcraft and Rift." In Proceedings of the International Conference on the Foundations of Digital Games (FDG'12), ACM, 97-104.

Bjarke, Liboriussen and Paul Martin. "Regional game studies.” In Game Studiesvol. 16, no. 1 (2016).

Bloomberg. "Fueled by WeChat, Female Gamers Power Tencent's \$3 Billion Hit.” Accessed Sep 20, 2018. https://www.bloomberg.com/news/articles/2017-07-31/fueled-bywechat-female-gamers-power-tencent-s-3-billion-hit

Blizzard Entertainment. Warcraft3[PC Computer]. Blizzard Entertainment, 2002. 
108 Effects of Mobile Platform on Female Engagement in MOBA Games

Bluehole. PlayerUnknown's Battlegrounds [PC Computer]. Bluehole. 2017.

Bonanno, Philip,and P.A.M. Kommers. "Gender differences and styles in the use of digital games.” In Educational Psychology vol. 25, no.1 (2005): 13-41.

Birk, M. and R. L. Mandryk. "Control your game-self: effects of controller type on enjoyment, motivation, and personality in game.”In Proceedings of the 31th SIGCHI conference on human factors in computing systems (CHI'2013). ACM, NewYork, 685-694.

Brehm, Audrey, L. "Navigating the feminine in massively multiplayer online games: gender in World of Warcraft." In Frontiers in Psychology vol. 4(2013): 903.

Brown, M., A. Kehoe, J. Kirakowski, and I. Pitt."Beyond the Gamepad: HCI and Game Controller Design and Evaluation.” In Evaluating User Experience in Games, edited by R. Bernhaupt, pp.263-285. London, UK: Springer, 2015.

Carr, Diane. "Contexts, gaming pleasures, and gendered preferences.” In Simulation \& Gaming: An Interdisciplinary Journal vol. 36(2005): 464-482.

Cashdan, Elizabeth. "Are men more competitive than women?" In British Journal of Social Psychology vol. 37, no. 2(1998): 213-229.

Cassell, J. and H. Jenkins. Chess for girls? Feminism and computer games, From Barbie to Mortal Kombat: gender and computer games. Cambridge, MA: MIT Press, 1998.

Consalvo, M. Atari to Zelda: Japan's Videogames in Global Contexts. Cambridge, MA: MIT Press, 2016. 
Effects of Mobile Platform on Female Engagement in MOBA Games 109

Corbin, J. and A. Strauss. Basics of qualitative research (4th Ed). Thousand Oaks, CA: Sage, 2015.

Downs, Edward and Stacy L. Smith. "Keeping abreast of hypersexuality: A video game character content analysis.” In Sex Roles vol. 62, no. 11-12(2010): 721-733.

Epic Games. Fortnite [PC Computer, PS4, XBOX]. Epic Games. 2017.

ESA.“ESA 2015 Annual Report.” Accessed Sep 20, 2018. http://www.theesa.com/wp-content/uploads/2016/04/ESAAnnual-Report-2015

ESA. “ESA 2017 Annual Report.” Accessed Sep 20, 2018. http://www.theesa.com/wp-content/uploads/2017/09/ESAAnnualReport-Digital-91917.pdf

Gao, G., A. Min, and P.C. Shih. "Gendered Design Bias: Gender Differences of In-Game Character Choice and Playing Style in League of Legends." In Proceedings of the 29th Australian Conference on Computer-Human Interaction (OzCHI'17), ACM, New York, 307-317.

Gerling, K. M., M. Klauser, and J. Niesenhaus. "Measuring the impact of game controllers on player experience in FPS games." In Proceedings of the 15th International Academic Mind Trek Conference: Envisioning Future Media Environments (MindTrek'11). ACM, NewYork, 83-86.

Gneezy, Uri, M. Niederle and A. Rustichini. "Performance in competitive environments: Gender differences.” In The Quarterly Journal of Economics, vol. 118, no. 3(2003):1049-1074.

Gray, Kishonna L. "Intersecting oppressions and online communities: Examining the experiences of women of color in XboxLive.” In Information, Communication \& Society vol. 15, no.3(2012): 411-428. 
110 Effects of Mobile Platform on Female Engagement in MOBA Games

Halloran, J., G. Fitzpatrick, Y. Rogers and P. Marshall. "Does it matter if you don't know who's talking? multiplayer gaming with voice over IP." In Proceedings of the extended abstracts on Human factors in computing systems (CHI EA'04), ACM, New York,1215-1218.

Hartmann,Tilo and Christoph Klimmt. “Gender and computer games: Exploring females'dislikes.” In Journal of Computer Mediated Communication vol. 11, no. 4(2006): 910-931.

Holtzblatt, K. and S. Jones. "Contextual Inquiry: A participatory technique for system design” In Participatory design: Principles and practices, edited by D. Schuler, A. Namioka, pp. 177-210. Hillsdale, New Jersey: Lawrence Erlbaum Associates, 1993.

Huffaker, D., J. Wang, J. Treem, M. A. Ahmad, L. Fullerton, D. Williams, M. S. Poole and N. Contractor. "The social behaviors of experts in massive multiplayer online role-playing games.” In Proceedings of Computational science and engineering International conference (CSE '09) vol. 4, IEEE, 326-331.

Inkpen, Kori, Rena Upitis, Maria Klawe, Joan Lawry, Ann Anderson, Mutindi Ndunda, Kamran Sedighian, Steve Leroux and David Hsu. “ 'We Have Never-Forgetful Flowers In Our Garden': Girl's Responses To Electronic Games.” In Journal of Computers in Mathematics and Science Teaching vol.13(1994): 383-383.

Innocent,T. and S. Haines. "Nonverbal communication in multiplayer game worlds.” In Proceedings of the 4th Australasian conference on Interactive entertainment (IE'07), RMIT University,11.

Jenson, Jen, and Suzanne de Castell. “Gender, simulation, and gaming: Research review and redirections.” In Simulation \& Gaming vol. 41, no.1(2010): 51-71.

Jiguang. “King of Glory Report.” Accessed Sep 20, 2018. https://www.jiguang.cn/reports/72 
Effects of Mobile Platform on Female Engagement in MOBA Games 111 King of Glory Homepage. Accessed Sep 20, 2018. http://pvp.qq.com/

League of Legends. Accessed Sep 20, 2018 .https://na.leagueoflegends.com/en/

Liu, Yong and Hongxiu Li. "Exploring the impact of use context on mobile hedonic services adoption: An empirical study on mobile gaming in China" In Computers in Human Behavior vol. 27, no. 2 (2011): 890-898.

Limperos, Anthony M., Michael G. Schmierbach, Andrew D. Kegerise and Frank E. Dardis. "Gaming across different consoles: exploring the influence of control scheme on game-player enjoyment.” In Cyberpsychology, Behavior, and Social Networking vol. 14, no. 6 (2011):345-350.

Lucas,Kristen, and John L. Sherry. "Sex differences in videogame play: A communication-based explanation.” In Communication Research vol.31 ,no.5 (2004):499-523.

Lindley, S. E., J. LeCouteur and N. L. Berthouze. "Stirring up experience through movement in gameplay: effects on engagement and social behaviour." In Proceedings of the 26th SIGCHI Conference on Human Factors in Computing Systems (CHI’08), ACM, NewYork, 511-514.

Martey, R.M., Jenny Stromer-Galley, Jaime Banks, Jingsi Wu and Mia Consalvo. "The strategic female: Gender-switching and player behavior in online games.” In Information, Communication \& Society vol. 17, no. 3(2014): 286-300.

McEwan, M., D. Johnson, P. Wyeth and A. Blackler. "Videogame control device impact on the play experience." In Proceedings of The 8th Australasian Conference on Interactive Entertainment: Playing the System (IE'12). ACM, 18. 
112 Effects of Mobile Platform on Female Engagement in MOBA Games

Moonton. Mobile Legends [Mobile Devices, Online Game]. Moonton, 2016. Played April 20, 2017.

Mou, Y., and W. Peng. "Gender and Racial Stereotypes in Popular Video Games.” In Handbook of Research on Effective Electronic Gaming in Education, edited by R. Ferdig, pp. 922-937. Hershey, PA: IGI Global, 2009.

Nakandala, Supun, G. L. Ciampaglia, N.M. Su and Y.Y. Ahn. “Gendered Conversation in a Social Game-Streaming Platform.”In Proceedings of the Eleventh International AAAI Conference on Web and Social Media (ICWSM' 2017). Montreal, Canada, 162-171.

Niederle, Muriel, and Lise Vesterlund. "Do women shy away from competition? Do men compete too much?” In The Quarterly Journal of Economics vol.122, no. 3(2007): 1067-1101.

Olson, Cheryl K, Lawrence A. Kutner and Dorothy E.Warner. "The role of violent videogame content in adolescent development: Boys' perspectives." In Journal of Adolescent Research vol.23,no.1(2008):55-75.

Peña, Jorge, Jeffrey T. Hancock and Nicholas A. Merola. “The priming effects of avatars in virtual settings." In Communication Research vol.36, no.6(2009): 838-856.

Penguin Intelligence. Accessed Sep 20, 2018. http://games.qq.com/a/20170808/008286.htm

Persky, Susan and Jim Blascovich."Immersive virtual environments versus traditional platforms: Effects of violent and nonviolent videogame play.” In Media Psychology vol.10(2007): 135-56.

Phan,M. H., J. R. Jardina, S. Hoyle and B. S. Chaparro. "Examining the role of gender in videogame usage, preference, 
Effects of Mobile Platform on Female Engagement in MOBA Games 113 and behavior." In Proceedings of the Human Factors and Ergonomics Society Annual Meeting vol.56 (2012), 1496-1500.

QuestMobile. "2018 Spring China Mobile Internet Report." $\begin{array}{llll}\text { Accessed } & \text { Sep } 2018 . & \text { (2018a). }\end{array}$ https://www.questmobile.com.cn/research/report-new/5

QuestMobile. “2017 Mobile Social Report.” Accessed Sep 20, 2018. (2018b). https://www.questmobile.com.cn/research/reportnew/16

Ratan, Rabindra A., Nicholas Taylor, Jameson Hogan, Tracy Kennedy and Dmitri Williams. "Stand by your man: An examination of gender disparity in League of Legends.” In Games and Culture vol. 10, no. 5 (2015): 438-462.

Richard, G., and C. M. Hoadley. "Investigating a supportive online gaming community as a means of reducing stereotype threat vulnerability across gender.” In Proceedings of Games, Learning \& Society vol. 9 (2013), 261-266.

Riot Games. League of Legends [PC Computer, OnlineGame]. Riot Games, 2009. Played 1 May 2018.

Rogers, Ryan, Nicholas David Bowman and Mary Beth Oliver. "It's not the model that doesn't fit, it's the controller! The role of cognitive skills in understanding the links between natural mapping, performance, and enjoyment of console video games." In Computers in Human Behavior vol.49(2015): 588-596.

Royse, Pam, Joon Lee, Baasanjav Undrahbuyan, Mark Hopson and Mia Consalvo. "Women and games: Technologies of the gendered self.” In New Media \& Society vol. 9, no. 4(2007): 555-576.

Salter, Anastasia, Bridget Blodgett (2012) "Hypermasculinity \& Dickwolves: The Contentious Role of Women in the New Gaming 
114 Effects of Mobile Platform on Female Engagement in MOBA Games

Public.” In Journal of Broadcasting \& Electronic Media vol. 56, no. 3(2012): 401-416

Schmierbach, Mike. “'Killingspree':Exploring the connection between competitive gameplay and aggressive cognition.” In Communication Research vol.37, no.2(2010): 256-274.

Schröder, Arne. “ 'We don't want it changed, do we?'-Gender and Sexuality in Role Playing Games.” In Journal for Computer Game Culture vol. 2, no. 2(2008): 241-256.

Shaer, O., L. Westendorf, N. A. Knouf and C. Pederson. "Understanding Gaming Perceptions and Experiences in a Women's College Community." In Proceedings of the 2017 Conference on Human Factors in Computing Systems (CHI'17), ACM, 1544-1557.

Shaw, Adrienne. "What is video game culture? Cultural studies and game studies.” In Games and culture vol. 5, no. 4 (2010): 403-424.

Super Evil Megacorp. Vainlory [MobileDevices]. Super Evil Megacorp, 2014.

Tang, Wai Yen and Jesse Fox. "Men's harassment behavior in online videogames: Personality traits and game factors.”In Aggressive Behavior vol.42, no.6(2016): 513-521.

Taylor, T. L. Play between worlds: Exploring online game culture.Cambridge, UK: MIT Press, 2009.

Taylor, Nicholas, Jen Jenson and Suzanne de Castell. "Cheerleaders/booth babes/Halo hoes: pro-gaming, gender and jobs for the boys." In Digital Creativity vol. 20, no. 4(2009): 239-252.

Tencent Games. King of Glory [Mobile Devices]. Tencent Games, 2015. Played Sep 1, 2018. 
Effects of Mobile Platform on Female Engagement in MOBA Games 115 Tencent Games. Arena of Valor [Mobile Devices].Tencent Games, 2018.

Todd, Cherie. “'Troubling’ gender in virtual gaming spaces.” In New Zealand Geographer vol. 68, no. 2(2012): 101-110.

Tychsen, A. "Role playing games: comparative analysis across two media platforms." In Proceedings of the 3rd Australasian conference on Interactive Entertainment (IE'06), Perth, Australia, 75-82.

Tychsen, Anders, Michael Hitchens and Thea Brolund.“Character play - the use of game characters in multi-player role-playing games across platforms.” In Computers in Entertainment (CIE) vol.6(2008), 22.

Umeng. "Research on mobile game users in 2017.” Accessed Sep 20, 2018. http://tip.umeng.com/uploads/data_report/ research_on_mobile_game_users_in_2017.pdf?mm_unid=1_1307 2_53050108390753576805095d6d03595202\&jlogid=012019585 51qn52d

Vermeulen, Lotte, Elena Núñez Castellar and Jan Van Looy. "Challenging the other: Exploring the role of opponent gender in digital game competition for female players.” In Cyberpsychology, Behavior, and Social Networking vol. 17, no. 5(2014): 303-309.

Vugt, Mark Van, David De Cremer and Dirk P. Janssen. “Gender differences in cooperation and competition: The male-warrior hypothesis.” In Psychological Science vol. 18, no. 1(2007): 19-23.

Yee, Nick. "7 Things We Learned About Primary Gaming Motivations From Over 250,000 Gamers,” Quantic Foundry, last modified 15 December 2016, http://quanticfoundry.com/2016/12/ 15/primary-motivations/

Yee, Nick. "Beyond 50/50: Breaking Down the Percentage of Female Gamers by Genre,” Quantic Foundry, last modified 19 
116 Effects of Mobile Platform on Female Engagement in MOBA Games

January 2017, http://quanticfoundry.com/2017/01/19/femalegamers-by-genre/

Yee, Nick. "Motivations for play in online games." In CyberPsychology \& Behavior vol. 9, no. 6(2006): 772-775.

Yee, Nick. and J. Bailenson. "The Proteus effect: The effect of transformed self- representation on behavior." In Human Communication Research vol. 33, no. 3(2007): 271-290.

Yee, Nick. "Maps of digital desires: Exploring the topography of gender and play in online games.” In Beyond Barbie and Mortal Kombat: New perspectives on gender and gaming, edited by Y. B. Kafai, C. Heeter, J. Denner, J. Y. Sun, pp. 83-96. Cambridge, MA: MIT Press, 2008.

Yee, Nick. The Proteus paradox: How online games and virtual worlds change us-and how they don't. New Haven, CT: Yale University Press, 2014.

Zynga.Solstice Arena[Mobile Devices]. Zynga, 2013. 